\title{
Single-shot color digital holography based on the fractional Talbot effect
}

\author{
María A. Araiza-Esquivel, ${ }^{1}$ Lluís Martínez-León, ${ }^{2,3, \star}{ }^{*}$ Bahram Javidi, ${ }^{4}$ \\ Pedro Andrés, ${ }^{5}$ Jesús Lancis, ${ }^{2,3}$ and Enrique Tajahuerce ${ }^{2,3}$ \\ ${ }^{1}$ Laboratorio de Procesamiento Digital de Señales, Universidad Autónoma \\ de Zacatecas, 98000 Zacatecas, Mexico \\ ${ }^{2}$ GROC.UJI, Departament de Física, Universitat Jaume I, 12071 Castelló, Spain \\ ${ }^{3}$ Institut de Noves Tecnologies de la Imatge, Universitat Jaume I, 12071 Castelló, Spain \\ ${ }^{4}$ Department of Electrical and Systems Engineering, University of Connecticut, \\ Storrs, Connecticut 06269-2157, USA \\ ${ }^{5}$ Department d'Òptica, Universitat de València, 46100 Burjassot, Spain \\ *Corresponding author: Iluis.martinez@uji.es
}

Received 30 July 2010; revised 10 December 2010; accepted 18 January 2011; posted 20 January 2011 (Doc. ID 132538); published 28 February 2011

\begin{abstract}
We present a method for recording on-axis color digital holograms in a single shot. Our system performs parallel phase-shifting interferometry by using the fractional Talbot effect for every chromatic channel simultaneously. A two-dimensional binary amplitude grating is used to generate Talbot periodic phase distributions in the reference beam. The interference patterns corresponding to the three chromatic channels are captured at once at different axial distances. In this scheme, one-shot recording and digital reconstruction allow for real-time measurement. Computer simulations and experimental results confirm the validity of our method. (C) 2011 Optical Society of America

OCIS codes: $\quad 090.1705,090.1995,070.6760,050.5080$.
\end{abstract}

\section{Introduction}

Digital holography is a convenient technique to record and reconstruct the complex amplitude distribution associated with diffracted light beams. Through digital recording and processing procedures, it has been possible to develop new systems for a variety of applications such as three-dimensional (3D) imaging, microscopy, interferometry, or information security [1]. In principle, phase-shifting techniques are among the most efficient in terms of spatial resolution to record digital holograms [2]. However, they generally require sequential acquisition of interference patterns with different phase retardations of the reference beam, which is what prevents real-time

0003-6935/11/070B96-06\$15.00/0

(C) 2011 Optical Society of America measurement. Several techniques for time-resolved dynamic interferometry have been developed allowing one-shot operation and acquisition times only limited by the sensor capabilities [3-7].

On the other hand, a number of digital holographic techniques deal with color objects by using either offaxis [8-10] or phase-shifting interferometry [11]. Color digital holography presents additional challenges than single wavelength operation, owing to the dependence on wavelength of light propagation and phase-shifting techniques. Nevertheless, as in monochromatic applications, several procedures for dynamic color digital holography have been proposed based on parallel phase-shifting techniques [12]. They allow capturing digital holograms from color objects in one-shot operations under polychromatic illumination. 
Recently, a new method for recording on-axis digital holograms in a single shot with parallel phaseshifting techniques has been presented by our group, and it has been confirmed by experimental results [13]. The proposal is based on the fractional Talbot effect provided by two-dimensional (2D) black-andwhite gratings [14]. Numerical simulations based on a related method that takes advantage, as well, of the Talbot effect for performing one-shot digital holography, has been reported $[15,16]$. However, unlike this proposal and many other parallel phaseshifting techniques, our approach does not require complex diffractive optical elements or periodic pixelated polarization devices (including retarders), which are difficult to build and hard to integrate with the CCD sensor.

In this contribution, we extend our technique to the recording and reconstruction of color digital holograms under polychromatic illumination. The system is based on the polychromatic fractional Talbot effect exhibited by a simple 2D binary amplitude grating. The grating codifies the reference beam of an interferometer and generates the same periodic phase distribution, with uniform irradiance, at a different axial distance for each spectral component of the incident light. Because of the properties of the Talbot effect, the technique intrinsically guarantees the proper phase shifting for every spectral component, though at different planes. This reference field interferes with the object diffraction pattern to be measured. In the proposed scheme, a modified 3-CCD color sensor records the interferogram for each chromatic component. By sampling and processing this color pixelated phase-shifted interferogram, we are able to measure the amplitude and phase of the object light field for each chromatic channel, thus allowing for time-resolved dynamic operation.

The remaining of the paper is organized as follows: In Section 2, we explain the basis of our Talbot color digital holography technique. Section 3 presents numerical simulations testing the capabilities of the method; whereas, experimental results are reported in Section 4. Finally, in Section $\underline{5}$, we summarize the conclusions of our work.

\section{Talbot Digital Holography with Polychromatic Light}

Parallel phase-shifting techniques offer single-shot recording by means of generating a phase distribution with at least three relative phase shifts in different cross-section areas of the reference beam. Our single-shot digital holography technique creates a periodic phase distribution in the reference beam on account of the fractional Talbot effect, so that the interference between the object beam and a threestep periodic phase modulation in the reference beam provides the information to retrieve the object complex amplitude distribution.

When a grating is illuminated by a monochromatic coherent parallel light beam, replicas of the grating appear periodically at distances multiple of the socalled Talbot distance. At fractions of the Talbot dis- tance, Fresnel images, the superposition of shifted replicas of the grating weighted by different phase factors, appear [14]. In principle, light diffraction by free-space propagation exhibits a strong dependence on the wavelength of the incident beam. However, under parallel polychromatic illumination, an amplitude $2 \mathrm{D}$ grating generates the same Fresnel image, and with the same scale, for each spectral component of the incident light, but located at a different axial distance. Fresnel images are obtained at distances

$$
z^{\prime}(\lambda)=\frac{2 d^{2}}{\lambda}\left(q+\frac{n}{m}\right)=z_{t}\left(q+\frac{n}{m}\right),
$$

where $d$ is the period of the grating, $\lambda$ is the wavelength corresponding to each chromatic channel, $z_{t}$ is the Talbot distance, $q$ is an integer, and $n$ and $m$ are natural numbers with no common factor. For achieving the simplest $2 \mathrm{D}$ periodic phase distribution with three phase steps, we consider $n / m=$ $1 / 4$ [13] together with an opening ratio of the binary grating of $1 / 4$. This Fresnel image exhibits uniform irradiance and a periodic distribution, with period $d$,

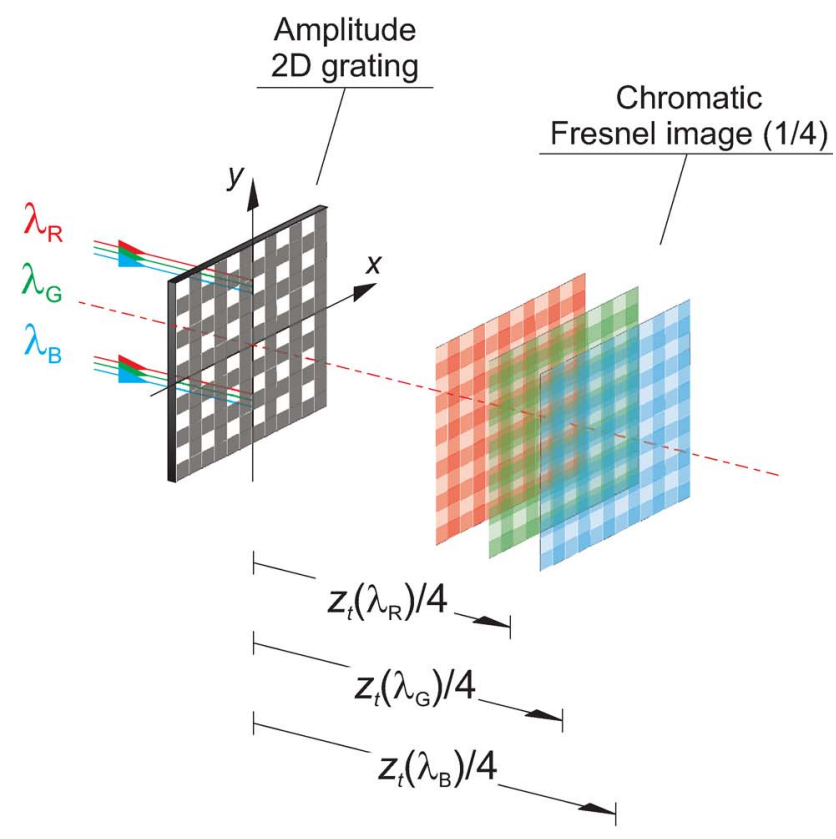

(a)

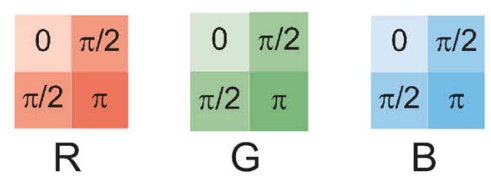

(b)

Fig. 1. (Color online) Generation of Fresnel images by a 2D amplitude grating under polychromatic illumination. (a) The corresponding Fresnel image for each spectral component presents the same scale, but is located at a different axial distance. (b) The relative phase distribution in the unit cell is the same for each chromatic channel. 
of different relative phases $0, \pi / 2$, and $\pi$. A similar Fresnel image is obtained with $n / m=3 / 4$. Thus, with the aid of the fractional Talbot effect, phase-only modulation is achieved in the reference beam. The Fresnel images associated with these parameters for the red (R), green (G), and blue (B) chromatic channels are shown in Fig. 1(a). The same periodic phase distribution with uniform irradiance is obtained for all the spectral components, although at different axial distances. Fig. 1(b) shows the values of the relative phase in the unit cell of the periodic phase distribution for the three wavelengths that provide the chromatic channels R, G, and B. Therefore, our technique provides the same phase-shifting steps $(0, \pi / 2$, and $\pi)$ for the three wavelengths by using a single element in a very simple way.

Figure 2 shows one of the possible configurations to perform Talbot digital holography with polychromatic light, a Mach-Zehnder interferometer. A polychromatic laser beam is spatially filtered, collimated, and split into two uniform plane waves. The object beam illuminates the input object, and the light scattered travels toward the 3-CCD detector through the second beam splitter. The reference beam is diffracted by the $2 \mathrm{D}$ binary amplitude grating providing a periodic three-step phase distribution by the fractional Talbot effect. A 3-CCD camera captures the interference for each chromatic channel simultaneously. Each sensor of the 3-CCD camera has to be shifted axially by a small amount, with respect to the others, in such a way that they record the same Fresnel image for the three chromatic components. Therefore, we record three Talbot phase-shifted interferograms in one shot, each one for a different chromatic component.

To obtain the digital hologram from the corresponding Talbot phase-shifted interferogram we proceed in the following way for each chromatic component, as reported in detail in [13]. First, we sample the recorded interferogram periodically at the locations with constant reference phase, according to the periodic phase distribution given by the Fresnel image. Second, for the three resulting images, one for each phase, we find, by interpolation, the values of the irradiance at the spatial positions without information. In this way we obtain three full phaseshifted interferograms, with relative phase $0, \pi / 2$, and $\pi$. Next, we combine these interferograms $I(x, y ; \Delta \varphi)$, with $\Delta \varphi=0, \pi / 2$, and $\pi$, through a phaseshifting algorithm to obtain the object complex amplitude distribution $O(x, y)$. It is worth remarking that this process has to be performed in parallel for the three chromatic channels. Because the Fresnel image of interest for each wavelength is located at a slightly different axial distance, the output plane position is different to the same extent for each channel. The phase-shifting algorithm employed for each chromatic channel obeys the following relation:

$$
\begin{aligned}
O(x, y)= & \frac{1}{4}\{I(x, y ; 0)-I(x, y ; \pi) \\
& +i[2 I(x, y ; \pi / 2)-I(x, y ; 0)-I(x, y ; \pi)]\} .
\end{aligned}
$$

Finally, we reconstruct the complex amplitude distribution generated by the color object numerically by back propagating each chromatic component of the digital hologram by the appropriate distance.

\section{Numerical Simulations}

We have carried out numerical simulations to check the validity of our single-shot method for recording and reconstructing color digital holograms. Three parallel light beams with wavelengths $\lambda=488$, 514.5 , and $647 \mathrm{~nm}$ were considered. They illuminated a 2D color object, the Universitat Jaume I (UJI) logo, located at a distance of $\sim 400 \mathrm{~mm}$ from the camera. The sensor is supposed to have three CCDs, each with $N_{x} \times N_{y}=1024 \times 1024$ pixels with a size of $\Delta x=\Delta y=9 \mu \mathrm{m}$. The 2D binary amplitude grating had an open ratio equal to 0.25 and a period of $d=18 \mu \mathrm{m}$, double of the pixel size, in both dimensions, and located in the reference beam of the interferometer. The exact distance from the grating to each CCD depends on the Talbot distance for each wavelength, according to Eq. (1). Talbot distances

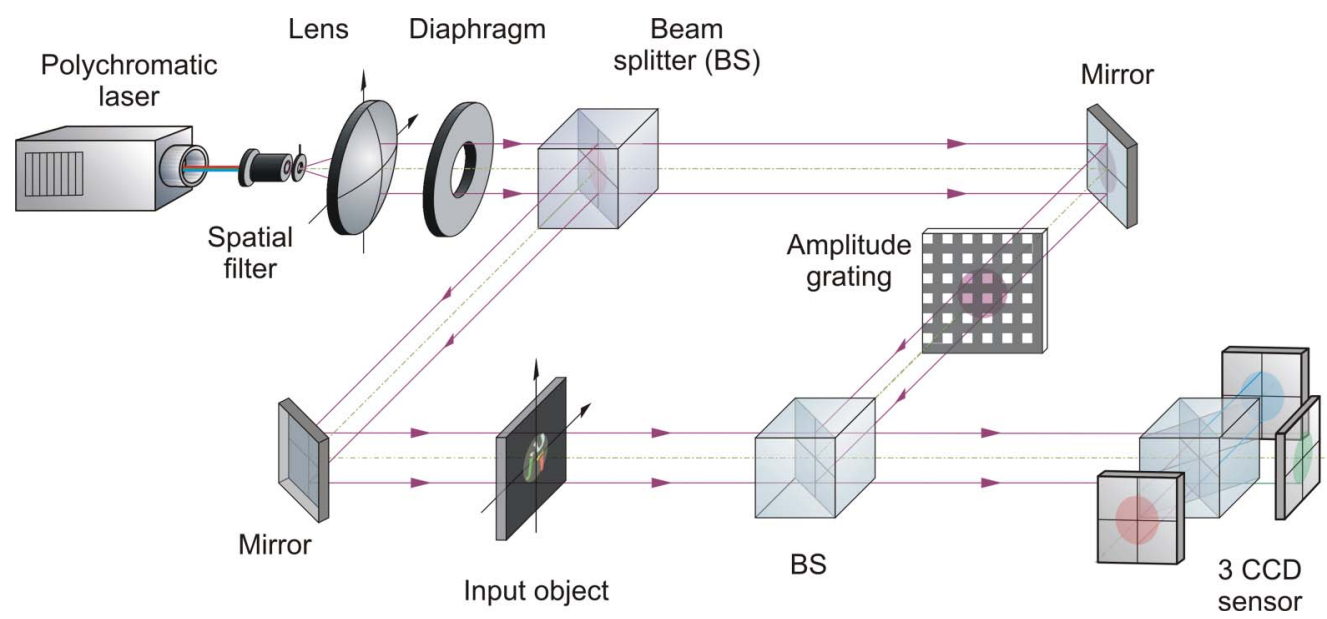

Fig. 2. (Color online) Setup for the recording of digital color holograms based on the fractional Talbot effect. 
are $z_{t}=1.33,1.26$, and $1.00 \mathrm{~mm}$, for $488,514.5$, and $647 \mathrm{~nm}$, respectively. We assumed $q=10$ and $n / m=3 / 4$. The diffraction patterns generated by the $2 \mathrm{D}$ object at the output planes and the Fresnel images generated by the grating were evaluated by using the propagation transfer function method. From the interference patterns of those fields, an interpolated interferogram was generated for each phase shift and for each chromatic channel. The digital holograms for each color were then determined using Eq. (2) and finally, the object diffraction patterns were reconstructed at their initial position. Figure 3 shows the process of hologram reconstruction. The UJI logo was decomposed in the R, G, and B channels, as displayed in Fig. 3(a). Figure 3(b) shows the irradiance distribution associated with each interferogram at the output plane of the Talbot color digital holography system, that is obtained by the interference between the Fresnel diffraction patterns of the 2D object and the Fresnel image of the grating. The holograms evaluated according to the phase-shifting algorithm are shown in Fig. 3(c). Figure 3(d) presents the reconstructed images of the objects obtained by computing the inverse Fresnel diffraction pattern from the corresponding digital hologram. Note that the object is clearly reconstructed.

The pixelated character of the recorded interferograms, due to the periodicity introduced by the fractional Talbot effect, can be observed in Fig. 4(a), where a magnified image of the highlighted area in Fig. $3(\mathrm{~b})$ is shown. Also, a magnified part of the green hologram, highlighted in Fig. 3(c), is displayed in Fig. 4(a), after interpolation and evaluation of the hologram through the phase-shifting algorithm.

Finally, Fig. 5 compares the initial object, the UJI logo. The original logo can be seen in Fig. 5(a). In Fig. 5(b) the color reconstruction, which has been synthesized from the three chromatic channel reconstructions, is shown. The simulated reconstruction is highly similar to the original object. Nevertheless, the reconstruction may be somehow impaired when more restrictive conditions apply. For instance, in some of the simulations, a random phase mask

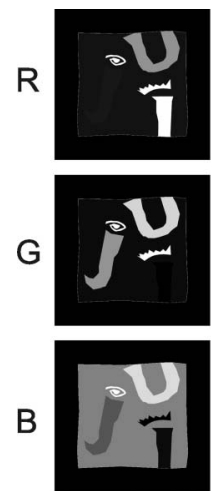

(a)
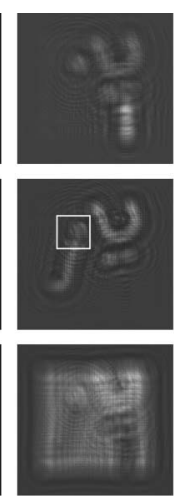

(b)

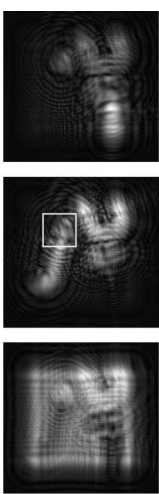

(c)

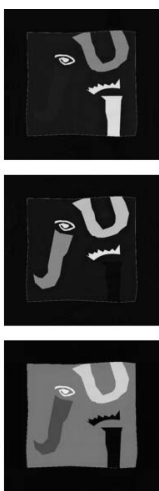

(d)
Fig. 3. Simulation of hologram reconstruction showing for each chromatic channel: (a) 2D object, (b) interferogram, (c) interpolated hologram, (d) reconstructed image.

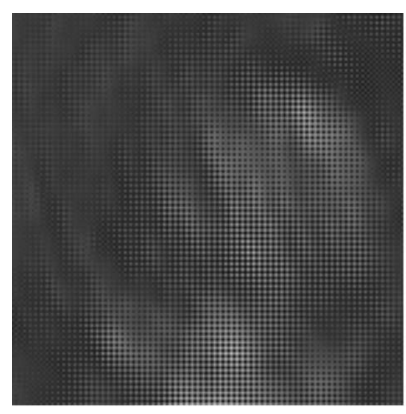

(a)

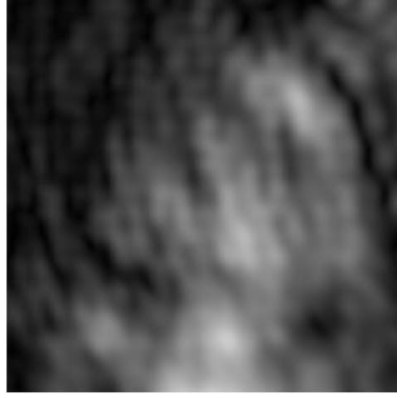

(b)
Fig. 4. Magnified image of the highlighted areas in Fig. $\underline{3}$, corresponding to the G channel, for (a) the interferogram (showing the pixelated structure) and (b) the interpolated hologram.

was attached to the binary images in order to spread the diffraction patterns at the output plane. In that case, the reconstruction obtained is presented in Fig. 5(c).

\section{Experimental Results}

To perform experiments for validating the method, we assembled a Talbot digital holographic system, like the setup shown in Fig. 2, using a Mach-Zehnder interferometer. The light source was an $\mathrm{Ar}-\mathrm{Kr}$ laser emitting three wavelengths with 488, 514.5, and $647 \mathrm{~nm}$, thus providing three chromatic channels. The amplitude mask was a binary square grating (with a size of $2 \mathrm{~cm} \times 2 \mathrm{~cm}$ and a period of $72 \mu \mathrm{m}$ ) manufactured by direct laser lithography on a chrome photomask. In our experiment, the period of the grating was as large as 8 times the sensor pixel size, in order to alleviate experimental restraints. In this way, each square of constant phase had a size of $36 \mu \mathrm{m} \times 36 \mu \mathrm{m}$, corresponding to $4 \times 4$ pixels of the camera. Therefore, the resolution of our final hologram (with only $512 \times 512$ complex values) is 4 times lower than the maximum achievable.

The object, the UJI logo, was printed in a color transparency. Instead of a 3-CCD color camera, we used a single monochromatic CCD camera with 4 Mpixel that operated sequentially for each laser line. The CCD camera was located at a slightly different axial distance for each chromatic component in order to record each interferogram in a proper way. Even if the three interferograms have been recorded sequentially, the results are entirely comparable to those achievable with a 3 -CCD camera

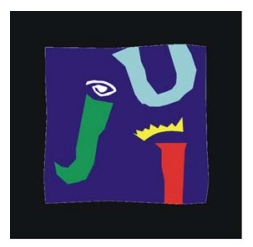

(a)

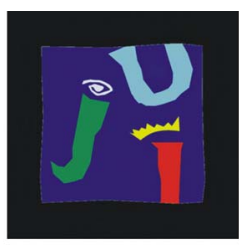

(b)

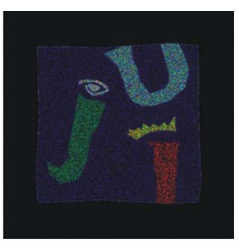

(c)
Fig. 5. (Color online) Simulation of hologram reconstruction. (a) Original color object. (b) Reconstructed object. (c) Reconstructed object when considering a random phase at the object plane. 


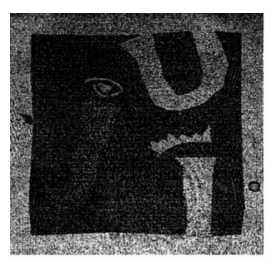

(a)

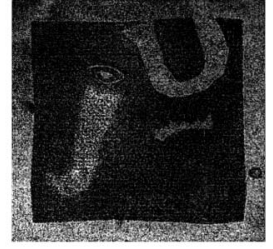

(b)

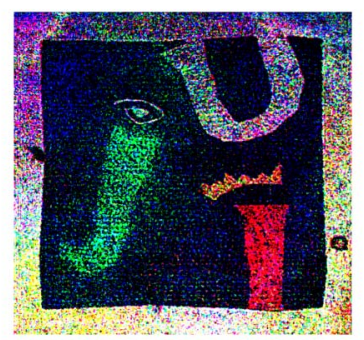

(d)

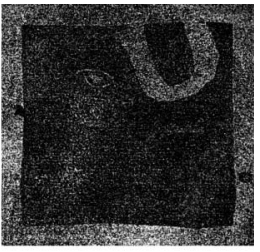

(c) three chromatic channels. (a) R. (b) G. (c) B. (d) Final reconstructed object.

in a single shot. By choosing a different Fresnel image index $q$ for some wavelengths, the axial distance between the phase distributions provided by the different wavelengths can be reduced. In fact, in our experiment, we chose $q=8, n / m=1 / 4$ for the B channel, but $q=8, n / m=3 / 4$ for the $\mathrm{G}$ channel, and $q=10, n / m=3 / 4$ for the $\mathrm{R}$ channel. Then, the $R$ channel sensor was located at $172.3 \mathrm{~mm}$ from the $2 \mathrm{D}$ grating and the $\mathrm{B}$ and $\mathrm{G}$ sensors were displaced +3 and $+4 \mathrm{~mm}$, respectively, with respect to the $\mathrm{R}$ channel sensor. Once the digital holograms were processed, images of the object were reconstructed evaluating the Fresnel diffraction integral in a computer, by using a fast Fourier transform algorithm for each chromatic component. The results are shown in Fig. 6. The reconstructed images for channels R, G, and $\overline{\mathrm{B}}$ are displayed in Figs. 6(a)-6(c), respectively. The synthesized color reconstruction is presented in Fig. 6(d). The quality of the reconstructed image is affected by both the limited resolution imposed by the period of the grating and the random phase introduced by the color transparency. These results confirm the validity of the approach, allowing single-shot color digital holography.

\section{Conclusions}

We have proposed a method for recording and reconstructing color holograms in a single shot by phaseshifting interferometry, using the periodic phase distribution provided by the fractional Talbot effect of a $2 \mathrm{D}$ grating. The approach, recently reported for a single wavelength, is extended to color systems owing to the chromatic properties of Fresnel images, i.e., the same periodic phase distribution in the reference beam is generated for different wavelengths, though in different axial positions. The simultaneous recording of the holograms corresponding to each chromatic channel can be accomplished with a mod- ified 3-CCD sensor. In our results, though, as a proof of principle, they have been recorded sequentially. The system assures direct color operation, and overcomes some classical complications of color digital holography. The time resolution for recording and reconstruction of color holograms with our method is only limited by the sensor acquisition time and the numerical processing, but it is independent of any factor inherent in our approach. Consequently, it allows dynamic operation with an in-line configuration. Furthermore, there is no need for special phase diffractive elements nor complex pixelated polarization devices, which is advantageous because miniature polarizing or retarding elements are difficult to build and hard to integrate with the CCD sensor. On the contrary, the codifying mask is very simple and is an off the shelf item. This element is not sensitive to the wavelength. In addition, the system does not depend on polarization.

Our method presents some limitations. As in the monochromatic version, the increase in time resolution is at the expense of a decrease in spatial resolution. However, this is common in most dynamic or parallel holographic methods based on spatial division multiplexing. Also, a fraction of light is lost by absorption in the periodic mask, but this can be an advantage in order to equalize the irradiance level of object and reference beams at the sensor plane.

The simulations and experiments presented in this paper were performed with $2 \mathrm{D}$ color objects. We will extend the system to 3D scenes in future work. Besides, the results could be further improved by using gratings with a smaller period that is better adapted to the sensor pixel size. Nevertheless, computer simulations and experimental results confirm the feasibility and flexibility of our method.

This work has been supported in part by the agreement between the Universitat Jaume I and the Fundació Caixa Castelló (Bancaixa), grant P1·1B2009-36. M. A. Araiza-Esquivel gratefully acknowledges a grant, 08I011.36/1 from the same agreement and partial support by PROMEP PIFI 2007-33-07, Mexico. B. Javidi is grateful for the support from the Guggenheim Foundation. The authors are very grateful to the Serveis Centrals d'Instrumentació Científica of the Universitat Jaume I for the use of the lithography system based on direct laser writing.

\section{References}

1. U. Schnars and W. P. O. Jüptner, "Digital recording and numerical reconstruction of holograms," Meas. Sci. Technol. 13, R85-R101 (2002).

2. I. Yamaguchi and T. Zhang, "Phase-shifting digital holography," Opt. Lett. 22, 1268-1270 (1997).

3. M. Novak, J. Millerd, N. Brock, M. North-Morris, J. Hayes, and J. Wyant, "Analysis of a micropolarizer array-based simultaneous phase-shifting interferometer," Appl. Opt. 44, 6861-6868 (2005).

4. Y. Awatsuji, A. Fujii, T. Kubota, and O. Matoba, "Parallel three-step phase-shifting digital holography," Appl. Opt. 45, 2995-3002 (2006). 
5. T. Nomura, S. Murata, E. Nitanai, and T. Numata, "Phaseshifting digital holography with a phase difference between orthogonal polarizations," Appl. Opt. 45, 4873-4877 (2006).

6. T. Nomura and M. Imbe, "Single-exposure phase-shifting digital holography using a random-phase reference wave," Opt. Lett. 35, 2281-2283 (2010).

7. B. Javidi, S. Yeom, and I. Moon, "Real-time 3D sensing, visualization and recognition of biological microorganisms," Proc. IEEE 94, 550-567 (2006).

8. P. Ferraro, S. Grilli, L. Miccio, D. Alfieri, S. De Nicola, A. Finizio, and B. Javidi, "Full color 3-D imaging by digital holography and removal of chromatic aberrations," J. Display Technol. 4, 97-100 (2008).

9. P. Tankam, Q. Song, M. Karray, J. Li, J. M. Desse, and P. Picart, "Real-time three-sensitivity measurements based on three-color digital Fresnel holographic interferometry," Opt. Lett. 35, 2055-2057 (2010).

10. K. Maejima and K. Sato, "One-shot digital holography for real-time recording of moving color 3-D images," in Digital Holography and Three-Dimensional Imaging, OSA Technical Digest Series (CD) (Optical Society of America, 2009), paper DMA2.
11. J. I. Kato, I. Yamaguchi, and T. Matsumura, "Multicolor digital holography with an achromatic phase shifter," Opt. Lett. 27, 1403-1405 (2002).

12. T. Kakue, T. Tahara, K. Ito, Y. Shimozato, Y. Awatsuji, K. Nishio, S. Ura, T. Kubota, and O. Matoba, "Parallel phase-shifting color digital holography using two phase shifts," Appl. Opt. 48, H244-H250 (2009).

13. Ll. Martínez-León, M. Araiza-E, B. Javidi, P. Andrés, V. Climent, J. Lancis, and E. Tajahuerce, "Single-shot digital holography by use of the fractional Talbot effect," Opt. Express 17, 12900-12909 (2009).

14. J. T. Winthrop and C. R. Worthington, "Theory of Fresnel images: I," J. Opt. Soc. Am. 55, 373-381 (1965).

15. A. Fajst, M. Sypek, M. Makowski, J. Suszek, and A. Kolodziejczyk, "Self-imaging phase mask used in digital holography with phase-shifting," Proc. SPIE 7141, 714123 (2008).

16. A. Siemion, M. Sypek, M. Makowski, J. Suszek, A. Siemion, D. Wojnowski, and A. Kolodziejczyk, "One-exposure phaseshifting digital holography based on the self-imaging effect," Opt. Eng. 49, 055802 (2010). 